\title{
Time Evolution, Correlations, and Linear Response of Non-Markov Processes *
}

\author{
P. Hänggi and $\mathrm{H}$. Thomas \\ Institut für Physik der Universität Basel, Basel, Switzerland \\ Received August 26, 1976
}

\begin{abstract}
We investigate the time evolution of stochastic non-Markov processes as they occur in the coarse-grained description of open and closed systems. We show that semigroups of propagators exist for all multivariate probability distributions, the generators of which yield a set of time-convolutionless master equations. We discuss the calculation of averages and time-correlation functions. Further, linear response theory is developed for such a system. We find that the response function cannot be expressed as an ordinary timecorrelation function. Some aspects of the theory are illustrated for the two-state process and the Gauss process.
\end{abstract}

\section{Introduction}

The stochastic behaviour of the coarse-grained variables of a system is described in general as a stochastic process with memory (non-Markov process) [1-3]. The Markov approximation, obtained by a coarsegraining in time, has been extensively used in the theory of collective phenomena far from thermodynamic equilibrium (see e.g. [4]). The interest in non-Markov behaviour has been renewed because of its relevance to certain non-equilibrium phase-transition phenomena $[5,6]$, and to transport problems in solids and fluids [7-9]. This question has been discussed in particular in context with the long-time behaviour of certain time-correlation functions in fluids $[8-10]$.

The stochastic properties of the system are characterized by a probability distribution $p(\mathbf{x} t)$ in the space $\Sigma$ of coarse-grained macrovariables $\mathbf{x}=\left(x_{1}, x_{2} \ldots\right)$ of the system. The microscopic degrees of freedom as well as the bath variables are eliminated by applying projector methods to the microscopic Liouville-von Neumann equation [1-3]. If the system is prepared at an initial time $t=t_{0}$ with probability distribution $p_{0}(\mathbf{x})$ without any correlations between the macrovariables $\mathbf{x}$ and the rest of the system, the rate of change of the probability distribution $p(t)$ is found

^ Work supported by the Swiss National Science Foundation to be given by the stochastic equation

$\frac{d p(t)}{d t}=\int_{t_{0}}^{t} \Lambda(t \mid s) p(s) d s$.

Here, the "retarded kernel" $\Lambda(t \mid s)$ is an operator acting on the space $\Pi$ of probability distributions ${ }^{\star}$. It describes the memory effect of the distribution at time $s$ on the rate of change at time $t$, and may contain a Markov contribution $\Lambda^{\text {Markov }}(t \mid s)=\Gamma(t) \delta\left(t-s-0^{+}\right)$. This equation has the formal solution

$p(t)=G\left(t \mid t_{0}\right) p_{0} \quad\left(t \geqq t_{0}\right)$

where the Green's function $G\left(t \mid t_{0}\right)$ is defined by the equation

$\frac{d G\left(t \mid t_{0}\right)}{d t}=\int_{t_{0}}^{t} \Lambda\left(t \mid t^{\prime}\right) G\left(t^{\prime} \mid t_{0}\right) d t^{\prime}$

with the initial condition $G\left(t_{0}^{+} \mid t_{0}\right)=\mathbb{1}$.

* Here and in the following, we use the following notation: $p(t)$ denotes an element of function space $\Pi$ with values $p(\mathbf{x} t)$. Elements like $\Lambda(t \mid s)$ are operators acting on $I$, with integral kernel $A(\mathbf{x} t \mid \mathbf{y} s)$ which may contain $\delta$-functions and their derivatives. Application of an operator to an element of function space, and operator multiplication are written in the usual way as products $\Lambda(t \mid s) p(s)$ and $\Lambda(t \mid s) \boldsymbol{A}\left(s \mid t_{1}\right)$, respectively:

$[\Lambda(t \mid s) p(s)]_{\mathbf{x}}=\int \Lambda(\mathbf{x} t \mid \mathbf{y} s) p(\mathbf{y} s) d y$

$\left[\Lambda(t \mid s) \Lambda\left(s \mid t_{1}\right)\right]_{\mathbf{x}, \mathbf{x}_{1}}=\int \Lambda(\mathbf{x} t \mid \mathbf{y} s) \Lambda\left(\mathbf{y} s \mid \mathbf{x}_{1} t_{1}\right) d y$ 
The Green's function $G\left(t \mid t_{0}\right)$ contains the effects of the initial preparation of the system. In most cases, however, one is interested in the behaviour of an aged system, in which the effects of initial preparation have died out. Therefore, the following problem suggests itself: Can one construct a general evolution equation

$p(t)=G(t \mid s) p(s) \quad(t \geqq s)$

for arbitrary times $t, s$ ? The existence of such an equation is not self-evident, because the corresponding equation for a Markov process is usually derived from the Chapman-Kolmogorov equation [11] which does not hold in the non-Markov case. In the present paper, this question is investigated in detail. We find that such an evolution equation always exists, independent of the validity of the Chapman-Kolmogorov equation. However, in contrast to the Markov case, the propagator $G(t \mid s)$ has in general not the significance of a conditional probability $R(t \mid s)$, with the exception of the solution $G\left(t \mid t_{0}\right)$ of Equation (1.3) relating back to the time $t_{0}$ of preparation of the system, which is indeed identical to the conditional probability $R\left(t \mid t_{0}\right)$. But neither $G(t \mid s)$ nor $G\left(t \mid t_{0}\right)$ can be used to compute joint probabilities $p^{(2)}(t, s)$ and time-correlation functions of an aged system. This restriction has not been observed in some recent work on non-Markov processes [12-15].

In Sections 2 and 3 we study the general problem of the time evolution of non-Markov systems. A set of stochastic equations for the rate of change of the singleevent and the multivariate distributions is derived, the solutions of which can be expressed in terms of propagators forming semigroups. These propagators must not be identified with the corresponding conditional probabilities. We also discuss the calculation of mean values and of time-correlation functions. Further, we investigate the asymptotic behaviour for large times. In Section 4, we present the linear-response theory for a Markovian perturbation. In contrast to the Markov case, the response tensor cannot be related by a generalized fluctuation theorem [16] to a stationary time-correlation function. Some aspects of the theory are illustrated in the Appendix for the twostate process and for the Gauss process.

\section{Time-Evolution of Single-Event Probability}

In this section, we investigate the basis for the existence of time-evolution equations of the form (1.4). In the case of a Markov process, there exists a conditional probability $R\left(\mathbf{x} t \mid \mathbf{x}_{1} t_{1}\right)$ with $t \geqq t_{1}$ which may be used for two different purposes: It is the integral kernel of the propagator $R\left(t \mid t_{1}\right)$ of the single-event probability $p(t)$, such that

$p(t)=R\left(t \mid t_{1}\right) p\left(t_{1}\right) \quad\left(t \geqq t_{1}\right)$, and it allows to compute the two-event joint probability

$p^{(2)}\left(\mathbf{x} t ; \mathbf{x}_{1} t_{1}\right)=R\left(\mathbf{x} t \mid \mathbf{x}_{1} t_{1}\right) p\left(\mathbf{x}_{1} t_{1}\right)$.

The simplicity of the Markov theory arises from the Chapman-Kolmogorov equation [11],

$R\left(t \mid t_{1}\right)=R(t \mid s) R\left(s \mid t_{1}\right) \quad\left(t \geqq s \geqq t_{1}\right)$

indicating that the elements $R\left(t \mid t_{1}\right)$ form a semigroup which can be generated from the infinitesimal propagator $R(t+d t \mid t)=\mathbb{1}+\Gamma(t) d t$ where

$\Gamma(t)=\left.\frac{d}{d s} R(s \mid t)\right|_{s=t^{+}}$.

In the non-Markov case, however, the conditional probability is a very complicated object, because it depends on previous history. The balance equation for the conditional probability

$R\left(t \mid t_{1}\right)=R\left(t \mid s, t_{1}\right) R\left(s \mid t_{1}\right)$

relates the time evolution of $R\left(t \mid t_{1}\right)$ to the higherorder conditional probability $R\left(t \mid s, t_{1}\right)$, and one can construct an infinite hierarchy of coupled evolution equations. This "memory" of the conditional probability, which is due to the fact that the sample functions passing through state $y$ at time $s$ remember which states they traversed at previous times, is illustrated in Figure 1. For the special case of an initially sharp distribution $p_{0}\left(x_{0}\right)=\delta\left(x_{0}-x_{0}^{\prime}\right)$, we find for instance

$R\left(x_{2} t_{2} \mid x_{1} t_{1}\right)$

$=\frac{\int R\left(x_{2} t_{2} \mid x_{1} t_{1}, x_{0} t_{0}\right) R\left(x_{1} t_{1} \mid x_{0} t_{0}\right) p_{0}\left(x_{0}\right) d x_{0}}{\int R\left(x_{1} t_{1} \mid x_{0} t_{0}\right) p\left(x_{0}\right) d x_{0}}$

$=R\left(x_{2} t_{2} \mid x_{1} t_{1}, x_{0}^{\prime} t_{0}\right)$

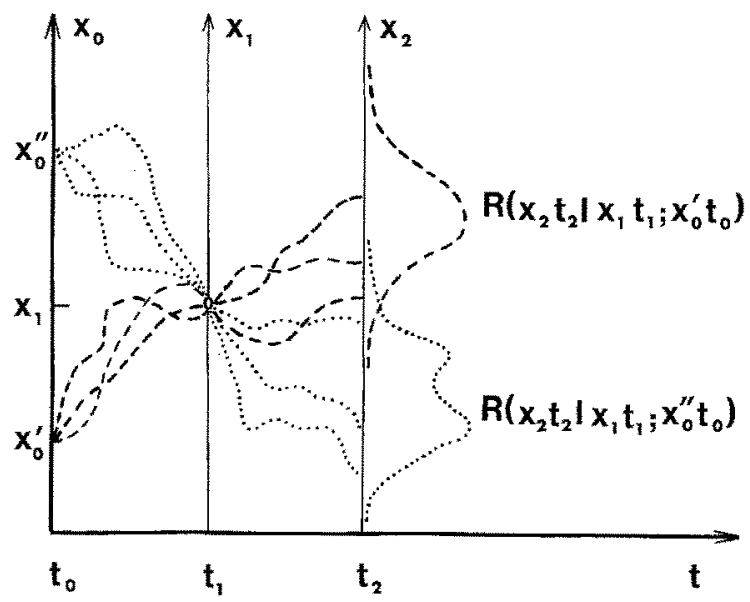

Fig. 1. Sample functions of a non-Markov process passing through $x_{1}$ at time $t_{1}$ remember at time $t_{2}$ which point $\left(x_{0}^{\prime}\right.$ or $x_{0}^{\prime \prime}$ ) they started from at time $t_{0}$. For a Markoy process, the two conditional distributions would be equal 
which shows that $R\left(t_{2} \mid t_{1}\right)$ depends on the initial state $x_{0}^{\prime}$. Note that $R\left(t \mid t_{0}\right)$, on the other hand, is always independent of the initial distribution $p_{0}$.

Thus, the elements $R(t \mid s)$ do not form a semigroup, and can therefore not be generated from an infinitesimal propagator. However, a significant simplification arises from the following theorem: Any stochastic process (Markov or non-Markov) satisfies

$p(t)=R(t \mid s) R\left(s \mid t_{1}\right) p\left(t_{1}\right)$.

Thus, although the Chapman-Kolmogorov Equation (2.3) does not hold for a non-Markov process, one obtains an equality if both sides are applied to the single-event probability $p\left(t_{1}\right)$. The proof of Equation (2.7) is evident from the definition of the conditional probability.

Using theorem (2.7) one may construct a whole class of semigroups of propagators $G\left(t \mid t_{1}\right)$ for the singleevent probability such that

$p(t)=G\left(t \mid t_{1}\right) p\left(t_{1}\right)$

with

$G\left(t^{+} \mid t\right)=\mathbb{1}$

and

$\int G\left(\mathbf{x} t \mid \mathbf{x}_{1} t_{1}\right) d x=1$.

The propagators $G\left(t \mid t_{1}\right)$ of each semigroup are the conditional probabilities of a Markov process which has the same single-event probabilities $p(t)$ (but different multivariate probabilities $p^{(n)}$ ) as the nonMarkov process under consideration.

The semigroup $G$ can be generated from the infinitesimal propagator $G(t+d t \mid t)=\mathbb{1}+\Gamma(t) d t$ with the generator $\Gamma(t)=d G(s \mid t) /\left.d s\right|_{s=t^{+}}$. The most obvious choice consists in using the conditional probability $R(t+d t \mid t)$ itself as infinitesimal propagator, whence

$\Gamma(t)=\left.\frac{d}{d s} R(s \mid t)\right|_{s=t^{+}}$.

However, this construction has the disadvantage that $R(s \mid t)$ depends on the initial distribution $p_{0}$ (see Fig. 1 and Eq. (2.6)). Thus, to every initial preparation of the system there will correspond a different substitutive Markov process. A propagator independent of $p_{0}$ is obtained by going back to the initial time $t_{0}$ at each infinitesimal step:

$\Gamma(t)=\left.\frac{d}{d s} R\left(s \mid t_{0}\right) R\left(t \mid t_{0}\right)^{-1}\right|_{s=t^{+}}$.

The significance of the time instant $t_{0}$ lies in the fact that at this time the system is prepared without any correlations between the macrovariables $\mathbf{x}$ and the rest of the system, i.e. without any memory of previous times $t<t_{0}$.

The generator $\Gamma(t)$ determines the rate of change of the single-event distribution by the time-convolutionless stochastic equation

$\frac{d p(t)}{d t}=\Gamma(t) p(t)$

and of the propagator by the "forward equation"

$\frac{d G\left(t \mid t_{1}\right)}{d t}=\Gamma(t) G\left(t \mid t_{1}\right)$

The formal solution of this differential equation can be written

$G\left(t \mid t_{1}\right)=\mathscr{T} \exp \int_{t_{1}}^{t} \Gamma(s) d s$

where $\mathscr{T}$ is the time-ordering operator. Equation (2.15) guarantees the semigroup property

$G\left(t \mid t_{1}\right)=G(t \mid s) G\left(s \mid t_{1}\right)$.

The propagator determined by the generator (2.12) has the simple properties

$G\left(t \mid t_{0}\right)=R\left(t \mid t_{0}\right)$

and

$G\left(t \mid t_{1}\right)=R\left(t \mid t_{0}\right) R\left(t_{1} \mid t_{0}\right)^{-1}$.

It can be obtained by Equation (1.3) in conjunction with the semigroup property (2.16) from the retarded kernel $A(t \mid s)$ which is directly related to the microscopic dynamics of the system.

The use of the reciprocal operator $R\left(t \mid t_{0}\right)^{-1}$ in (2.12) and (2.18) requires some comments. In the case of a finite number of states, the probability distributions are ordinary vectors and the operators are ordinary matrices, and there will be no problem to define $G^{-1}$ for a "connected" system. For a countable set of states, however, there is the possibility that the eigenvalues of $G$ converge to zero, and for a continuous state space, $G$ will in general have a continuous spectrum extending down to $0^{+}$. In these cases, $G^{-1}$ will not exist for all functions of function space $\Pi$. However, $G^{-1}\left(t \mid t_{1}\right)$ will always exist for functions $p(t)$ which have evolved out of a probability distribution $p\left(t_{1}\right)$, and only to such functions will operators like (2.12) and (2.18) be applied.*

With the help of the propagator $G\left(t \mid t_{1}\right)$, the statistical expectation of any state function $\phi(t)$ can be calculated from the statistical distribution $p\left(t_{1}\right)$ at an arbitrary fixed time $t_{1} \leqq t$,

$\langle\phi(\mathbf{x} t)\rangle=\iint \phi(\mathbf{x}, t) G\left(\mathbf{x} t \mid \mathbf{x}_{1} t_{1}\right) p\left(\mathbf{x}_{1}, t_{1}\right) d x_{1} d x$.

* The same remark applies to the use of the reciprocal propagator $R\left(t \mid t_{0}\right)^{-1}$ of a Markov process in Reference 16 
This includes all averages $\left\langle x_{\alpha}(t)\right\rangle$ of the state variables, all moments $\left\langle x_{\alpha}^{n}(t)\right\rangle$, and all equal-time correlations $\left\langle x_{\alpha}^{m}(t) x_{\beta}^{n}(t)\right\rangle$.

$\mathrm{Up}$ to this point, we have made no assumption about the behaviour against time-translations. We now assume time-translation invariance of the underlying microscopic process. It is important to note that even in this case the process is always nonstationary, in contrast to the Markov case. This is so because the effects of the preparation at time $t_{0}$ influence the evolution of the system for a finite time. Time-translation symmetry requires invariance with respect to a shift of all times, including $t_{0}$. Consequently, the single-event probability depends on $t-t_{0}$, and equations like (2.8) should be written more fully as

$p\left(t-t_{0}\right)=G\left(t-t_{0} \mid t_{1}-t_{0}\right) p\left(t_{1}-t_{0}\right)$

in order to show the dependence on $t_{0}$ explicitely. This nonstationarity which is due to the breaking of time-translation symmetry by the initial preparation of the system, should be distinguished from an explicitly nonstationary process controlled by timedependent external parameters, such as has been discussed for the Markov case in Reference 16.

One expects that in systems of physical interest the effects of initial preparation die out after some time, and the distribution tends to a stationary distribution

$p_{\text {as }}=\lim _{t_{0} \rightarrow-\infty} p\left(t-t_{0}\right)$

independent of the initial distribution $p_{0}$. This asymptotic stability of the single-event distribution is a consequence of the general ergodicity requirement discussed in Section 3. In the case of definition (2.11), there will exist a stationary asymptotic propagator

$G_{a s}\left(t-t_{1}\right)=\lim _{t_{0} \rightarrow-\infty} G\left(t-t_{0} \mid t_{1}-t_{0}\right)$

which has $p_{\text {as }}$ as eigenvector. In the case of definition (2.12), on the other hand, $G(t-\infty)$ exists and represents a time-independent singular operator with kernel

$G(\mathbf{x} t \mid \mathbf{y},-\infty)=R(\mathbf{x} t \mid \mathbf{y},-\infty)=p_{\text {as }}(\mathbf{x})$.

However, no such statements can be made for $G_{\text {as }}\left(t \mid t_{1}\right)$ in case of (2.12) and for $G(t \mid-\infty)$ in case of (2.11).

We have thus obtained an understanding of the existence of a propagator for the single-event probability in the non-Markov case. We stress again that the propagator $G\left(t \mid t_{1}\right)$ is not identical with the conditional probability $R\left(t \mid t_{1}\right)$ except for $t_{1}=t_{0}$ in the case of definition (2.12).

\section{Multivariate Distributions and Time-Correlation Functions}

The considerations of Section 2 can be generalized easily to the multivariate case. The multivariate dis- tribution $p^{(n)}(\mathbf{X} T)$ for the sequence of states $\mathbf{X} \equiv$ $\left(\mathbf{x}^{(1)} \ldots \mathbf{x}^{(n)}\right)$ at the time sequence $T \equiv\left(t^{(1)} \ldots t^{(n)}\right)$ is an element $p^{(n)}(T)$ of $\Pi^{n}$, and the conditional probability $R^{(n)}\left(\mathbf{X} T \mid \mathbf{X}_{1} T_{1}\right)$ is the kernel of an operator $R^{(n)}\left(T \mid T_{1}\right)$ acting on $\Pi^{n}$ such that

$p^{(n)}(T)=R^{(n)}\left(T \mid T_{1}\right) p^{(n)}\left(T_{1}\right)$,

where the smallest $t \in T$ is assumed to be larger than or equal to the largest $t_{1} \in T_{1}$, symbolically $T \geqq T_{1}$. For a non-Markov process, the balance equation

$R^{(n)}\left(T \mid T_{1}\right)=R^{(n)}\left(T \mid S, T_{1}\right) R^{(n)}\left(S \mid T_{1}\right)$

relates the time evolution of $R^{(n)}\left(T \mid T_{1}\right)$ to the higherorder conditional probability $R^{(n)}\left(T \mid S, T_{1}\right)$. Thus, the elements $R^{(n)}\left(T \mid T_{1}\right)$ do not form a semigroup and can therefore not be generated from an infinitesimal propagator. The simplification arises from the generalization of theorem (2.7): Any stochastic process satisfies

$p^{(n)}(T)=R^{(n)}(T \mid S) R^{(n)}\left(S \mid T_{1}\right) p^{(n)}\left(T_{1}\right)$.

This shows that $n$-parameter semigroups of propagators $G^{(n)}\left(T \mid T_{1}\right)$ for the multivariate distribution $p^{(n)}(T)$ can be constructed such that

$p^{(n)}(T)=G^{(n)}\left(T \mid T_{1}\right) p^{(n)}\left(T_{1}\right)$.

The semigroup $G^{(n)}$ can be generated from the vector generator

$\Gamma^{(n)}(T)=\left.\frac{\partial}{\partial S} G^{(n)}(S \mid T)\right|_{S=T^{+}}$

$\frac{\partial}{\partial S} \equiv\left(\frac{\partial}{\partial s^{(1)}}, \ldots, \frac{\partial}{\partial s^{(n)}}\right)$,

and there is again a multitude of choices for $\Gamma^{(n)}(T)$. One can generate the semigroup as in (2.11) by taking the conditional probability $R^{(n)}(T+d T \mid T)$ itself as infinitesimal propagator,

$\Gamma^{(n)}(T)=\left.\frac{\partial}{\partial S} R^{(n)}(S \mid T)\right|_{S=T^{+}}$,

but this has the disadvantage that it depends on the initial distribution $p_{0}$. In order to obtain a semigroup independent of the initial distribution, one has to relate back to the initial time $t_{0}$ as in (2.12),

$\Gamma^{(n)}(T)=\left.\frac{\partial}{\partial S} R^{(n)}\left(S \mid t_{0} D\right) R^{(n)}\left(T \mid t_{0} D\right)^{-1}\right|_{S=T^{+}}$

where $D=(1,1 \ldots 1)$ such that $t_{0} D$ denotes the $n$-tupel of initial times $\left(t_{0}, \ldots t_{0}\right)$.

The stochastic equation

$\frac{\partial}{\partial T} p^{(n)}(T)=\Gamma^{(n)}(T) p^{(n)}(T)$ 
can be integrated along a curve $T=T(\theta)$ in the space of $n$ times $T=\left(t^{(1)}, \ldots t^{(n)}\right)$ passing through the points $T_{1}=T\left(\theta_{1}\right)$ and $T_{2}=T\left(\theta_{2}\right)$ to give a solution of the form (3.4) with

$$
G^{(n)}\left(T_{2} \mid T_{1}\right)=\mathscr{T} \exp \int_{\theta_{1}}^{\theta_{2}} \Gamma^{(n)}(T(\theta)) \cdot \frac{d T(\theta)}{d \theta} d \theta
$$

where the ordering operator $\mathscr{T}$ acts on the curve parameter $\theta$. The semigroup property

$G^{(n)}\left(T \mid T_{1}\right)=G^{(n)}(T \mid S) G^{(n)}\left(S \mid T_{1}\right)$

follows immediately from (3.9) by choosing a curve which passes through $S$.

By adopting the definition (3.7) one obtains a propagator with the simple properties

$G^{(n)}\left(T \mid t_{0} D\right)=R^{(n)}\left(T \mid t_{0} D\right)$

and

$G^{(n)}\left(T \mid T_{1}\right)=R^{(n)}\left(T \mid t_{0} D\right) R^{(n)}\left(T_{1} \mid t_{0} D\right)^{-1}$.

The use of the reciprocal operator in (3.7) and (3.12) is subject to the same remarks as stated after Equation (2.18). In contrast to the single-event case, no method has been worked out yet to compute the multivariate distribution from the microscopic dynamics of the system.

With the help of the $n$-event propagator $G^{(n)}\left(T \mid T_{1}\right)$ one can calculate the $n$-event joint probability $p^{(n)}(T)$ and therefore any $n$-time correlation function from the single-event probability at an arbitrary fixed time $t_{1} \leqq T$. Because of $p^{(n)}\left(\mathbf{X}_{1}, t_{1} D\right)=p^{(1)}\left(\mathbf{x}_{1} t_{1}\right) \delta\left(\mathbf{x}_{1}^{(2)}-\mathbf{x}_{1}\right) \ldots$ $\delta\left(\mathbf{x}_{1}^{(n)}-\mathbf{x}_{1}\right)$ one finds

$p^{(n)}(\mathbf{X} T)=\int G^{(n)}\left(\mathbf{X} T \mid \mathbf{x}_{1} D, t_{1} D\right) p^{(1)}\left(\mathbf{x}_{1} t_{1}\right) d x_{1}$

and

$$
\begin{aligned}
& \left\langle\phi_{1}\left(\mathbf{x}^{(1)} t^{(1)}\right) \ldots \phi_{n}\left(\mathbf{x}^{(n)} t^{(n)}\right)\right\rangle \\
& =\int \phi_{1}\left(\mathbf{x}^{(1)}, t^{(1)}\right) \ldots \phi_{n}\left(\mathbf{x}^{(n)}, t^{(n)}\right) \\
& \times G^{(n)}\left(\mathbf{X} T \mid \mathbf{x}_{1} D, t_{1} D\right) p^{(1)}\left(\mathbf{x}_{1} t_{1}\right) d^{n} X d x_{1} .
\end{aligned}
$$

If the $n$-event propagator $G^{(n)}$ is known, all lower-order propagators can be calculated. It is not difficult to prove that the $(n-1)$-event propagator $\left.G^{(n-1}\right)$ can be obtained from $G^{(n)}$ by the same procedure as the $(n-1)$ event conditional probability $R^{(n-1)}$ is obtained from $R^{(n)}:$ In order to eliminate the $v$-th event from $G(\mathbf{X} T$ $\left.\mathbf{X}_{1} T_{1}\right)$, set $t^{(\nu)}=t^{(\mu)}, t_{1}^{(\nu)}=t_{1}^{(\mu)}, \mathbf{x}_{1}^{(\nu)}=\mathbf{x}_{1}^{(\mu)}$, where $t^{(\mu)}$ and $t_{1}^{(\mu)}$ are time instants adjacent to $t^{(\nu)}$ and $t_{1}^{(\nu)}$, respectively, and integrate over $\mathbf{x}^{(v)}$. We illustrate this procedure for the case $n=2$ :

$\int G^{(2)}\left(\mathbf{x}^{(1)} t^{(1)}, \mathbf{x}^{(2)} t^{(1)} \mid \mathbf{x}_{1}^{(1)} t_{1}^{(1)}, \mathbf{x}_{1}^{(1)} t_{1}^{(1)}\right) d x^{(2)}$

$=G^{(1)}\left(\mathbf{x}^{(1)} t^{(1)} \mid \mathbf{x}_{1}^{(1)} t_{1}^{(1)}\right)$.
It is also possible to keep all times of the sequence $T$ except the latest time $t^{(1)}$ fixed, and study propagation with respect to this $t^{(1)}$ alone. The corresponding propagator can be viewed as an operator acting on $\Pi^{1}$ which depends parametrically on the other events. Time-translation symmetry of the underlying microscopic process requires invariance with respect to a shift of all times, including $t_{0}$. Consequently, the multivariate distributions depend on $T-t_{0} D$, and this dependence can be exhibited explicitly by writing equations like (3.4) in the form

$p^{(n)}\left(T-t_{0} D\right)=G^{(n)}\left(T-t_{0} D \mid T_{1}-t_{0} D\right) p^{(n)}\left(T_{1}-t_{0} D\right)$.

In systems of physical interest, the effects of initial preparation of the system are expected to die out after some time. We call the process ergodic if

$\lim _{t_{0} \rightarrow-\infty} p^{(n)}\left(T-t_{0} D\right)=p_{\mathrm{as}}^{(n)}(T)$

exists for all $n$ and is independent of the initial preparation. Then, in the case of definition (3.7), $G^{(n)}(T \mid-\infty D)$ exists and represents a singular operator

$G^{(n)}(\mathbf{X} T \mid \mathbf{Y},-\infty)=p_{\text {as }}^{(n)}(\mathbf{X} T)$.

In the case of (3.6), no statement can be made on $G^{(n)}(T \mid-\infty D)$.

Time-translation symmetry requires that the process is asymptotically stationary, i.e. that $p_{\mathrm{as}}^{(n)}(T)$ is invariant under a shift of time

$p_{\mathrm{as}}^{(n)}(T+\tau D)=p_{\mathrm{as}}^{(n)}(T)$.

In the case of definition (3.6), there will exist an asymptotic propagator $G_{\text {as }}^{(n)}\left(T \mid T_{1}\right)$ which is stationary in the sense

$G_{\mathrm{as}}^{(n)}\left(T+\tau D \mid T_{1}+\tau D\right)=G_{\mathrm{as}}^{(n)}\left(T \mid T_{1}\right)$,

and $p_{\mathrm{as}}^{(n)}(T)$ will be eigenvector of $G_{\mathrm{as}}^{(n)}(T+\tau D \mid T)$. In the case of (3.7), no statement can be made on $G_{\text {as }}^{(n)}\left(T \mid T_{1}\right)$.

\section{Linear-Response Theory}

In this Section, we study the linear response of the system to an external perturbation. The perturbed single-event distribution $\hat{p}(t)$ satisfies the stochastic equation

$\frac{d \hat{p}(t)}{d t}=\int_{t_{0}}^{t} \hat{\Lambda}(t \mid s) \hat{p}(s) d s$

with the kernel

$\hat{\Lambda}(t \mid s)=\Lambda(t-s)+\Lambda^{\mathrm{ext}}(t \mid s)$.

Here, we assume that the unperturbed system is timetranslation invariant, such that its Green's function 
determined by Equation (1.3) depends only on the time difference $t-t_{0}$,

$G\left(t \mid t_{0}\right) \equiv R\left(t \mid t_{0}\right)=G\left(t-t_{0}\right)$.

The stochastic equation (4.1) is equivalent to the integral equation

$\hat{p}(t)=p(t)+\int_{t_{0}}^{t} G(t-s) \int_{t_{0}}^{s} \Lambda^{\mathrm{ext}}\left(s \mid s^{\prime}\right) \hat{p}\left(s^{\prime}\right) d s^{\prime} d s$.

From this equation, the perturbed distribution can be found by iteration. The first-order change is obtained by replacing $\hat{p}\left(s^{\prime}\right)$ by $p\left(s^{\prime}\right)$ under the integral.

For the further discussion we assume that the perturbation can be described by a Markov kernel, and write the perturbation in terms of time-dependent external forces $F_{i}(t)$,

$\Lambda^{\mathrm{ext}}(t \mid s)=\sum_{i} F_{i}(t) \Omega_{i} \delta\left(t-s^{+}\right)=\mathbf{F}(t) \cdot \mathbf{\Omega} \delta\left(t-s^{+}\right)$

where the $\Omega_{i}$ are linear operators acting on $\Pi$. Then, the first-order change of the single-event distribution is given by

$\delta \hat{p}(t)=\int_{t_{0}}^{t} G(t-s) \mathbf{F}(s) \cdot \mathbf{\Omega} p(s) d s$.

The linear-response tensor $\chi(t \mid s)$ is defined by the relation of the response of the state variables

$$
\begin{aligned}
& \langle\delta \mathbf{x}(t)\rangle \equiv\langle\mathbf{x}(t)\rangle^{\text {perturbed }}-\langle\mathbf{x}(t)\rangle^{\text {unperturbed }} \\
& =\int \mathbf{x} \delta \hat{p}(\mathbf{x} t) d x
\end{aligned}
$$

to the external forces,

$$
\langle\delta \mathbf{x}(t)\rangle=\int_{t_{0}}^{t} \chi(t \mid s) \cdot \mathbf{F}(s) d s
$$

We find

$$
\chi(t \mid s)=\theta(t-s) \iint \mathbf{x} \phi(\mathbf{y} s) G(\mathbf{x}, \mathbf{y} ; t-s) p(\mathbf{y} s) d x d y
$$

where we have introduced the vector-valued state function $\phi$ as in Reference 16

$\boldsymbol{\phi}(\mathbf{x} t)=[\mathbf{\Omega} p(t)]_{\mathbf{x}} / p(\mathbf{x} t)$.

Since the perturbation cannot change the normalization of the system, $\phi(\mathbf{x} t)$ has zero average,

$$
\langle\boldsymbol{\phi}(\mathbf{x} t)\rangle=\int[\mathbf{\Omega} p(t)]_{\mathbf{x}} d x=0,
$$

and represents thus a fluctuation (in general nonlinear) from the unperturbed state. Therefore, the state variable $\mathbf{x}$ may be replaced by its fluctuation $\boldsymbol{\xi}=\mathbf{x}-\langle\mathbf{x}\rangle^{\text {unperturbed }}$ in Equation (4.9).

It is important to note that in Equation (4.6) the perturbation $\mathbf{F}(s) d s$ applied at time $s$ is not, as one might have expected, propagated to time $t$ by the propagator
$G(t \mid s)$ corresponding to this time intervall, but rather by the propagator $G(t-s) \equiv G\left(t_{0}+t-s \mid t_{0}\right)$, i.e. in the same way as an initial distribution is propagated from $t_{0}$ to $t_{0}+t-s$. For the same reason, in contrast to the Markov case, the response tensor (4.9) is not given as a true two-time correlation function (generalized fluctuation theorem), but as a pseudo-correlation function

$\chi(t \mid s)=\theta(t-s) \ll \xi(t) \phi(\mathbf{y} s) 》$

calculated with a joint probability

$p_{2}^{\text {pseudo }}(\mathbf{x} t, \mathbf{y} s)=G(\mathbf{x}, \mathbf{y} ; t-s) p(\mathbf{y} s)$

which is the joint probability $p_{2}\left(t_{0}+t-s, t_{0}\right)$ of a system which was started with initial distribution $p(s)$.

The result obtained is the response of a nonstationary process, because it still contains the effects of initial preparation of the system. In the case of an ergodic process, the response of an aged system is obtained by performing the limit $t_{0} \rightarrow-\infty$. Then, $p(s)$ is replaced by $p_{\text {as }}$ in Equations (4.6) and (4.9), $\boldsymbol{\phi}(\mathbf{x} t)=\left[\boldsymbol{\Omega} p_{\text {as }}\right]_{\mathbf{x}} /$ $p_{\mathrm{as}}(\mathbf{x}) \equiv \boldsymbol{\phi}(\mathbf{x}(t))$ does not depend explicitly on time, and the response becomes stationary

$\chi_{\mathrm{as}}(t-s)=\theta(t-s)\langle\boldsymbol{\xi}(t) \boldsymbol{\phi}(\mathbf{y}(s))\rangle$.

We stress again that it is important to distinguish the propagator $G(t-s)$ determining the linear response from the asymptotic conditional probability $R_{\text {as }}(t-s)$ determining time-correlation functions of an aged system.

\section{Conclusions}

We have shown that semigroups of propagators $G^{(n)}$ for the $n$-time multivariate distributions $p^{(n)}$ exist for all $n$, which can be used to calculate averages and $m$-time correlation functions up to $m=n$ from the single-event distribution at an arbitrary fixed time. By going to the continuum limit, one may define a propagator $G(\mathbf{x}(t) \mid \mathbf{y}(s))$ which expresses the probability for a path $\mathbf{x}(t)$ as a path integral over paths $\mathbf{y}(s)$. The propagators $G^{(n)}$ of non-Markov processes are different from the conditional probabilities $R^{(n)}$ (except for conditions referring to the initial time $t_{0}$ ), and can therefore not be used to calculate $m$-time joint probabilities and correlation functions with $m>n$. The response to an external perturbation is given by the single-event propagator $G\left(t_{0}+\tau \mid t_{0}\right)$, and can therefore not be expressed in the form of an ordinary generalized fluctuation theorem.

\section{Appendix A. Two-State-Process}

The single-event distribution and the conditional probability of a general two-state process can be 
written in the form

$$
p(t)=\frac{1}{2}\left(\begin{array}{l}
1+a(t) \\
1-a(t)
\end{array}\right), \quad|a(t)| \leqq 1
$$

and

$$
\begin{gathered}
R\left(t \mid t_{1}\right)=\frac{1}{2}\left(\begin{array}{ll}
1+\rho\left(t, t_{1}\right)+\alpha\left(t, t_{1}\right) & 1-\rho\left(t, t_{1}\right)+\alpha\left(t, t_{1}\right) \\
1-\rho\left(t, t_{1}\right)-\alpha\left(t, t_{1}\right) & 1+\rho\left(t, t_{1}\right)-\alpha\left(t, t_{1}\right)
\end{array}\right) \\
\left|\rho\left(t, t_{1}\right) \pm \alpha\left(t, t_{1}\right)\right| \leqq 1,
\end{gathered}
$$

respectively. The time evolution of the single-event distribution is given by

$a(t)=\alpha\left(t, t_{1}\right)+\rho\left(t, t_{1}\right) a\left(t_{1}\right)$.

The conditional probabilities (A.2) do not form a semigroup except in the Markov case

$\rho\left(t, t_{1}\right)=\rho(t, s) \rho\left(s, t_{1}\right)$

$\alpha\left(t, t_{1}\right)=\alpha(t, s)+\rho(t, s) \alpha\left(s, t_{1}\right)$.

The generator of any semigroup has the form

$\Gamma(t)=\frac{1}{2}\left(\begin{array}{rr}\dot{\mathrm{P}}(t)+\dot{\mathrm{A}}(t) & -\dot{\mathrm{P}}(t)+\dot{\mathrm{A}}(t) \\ -\dot{\mathrm{P}}(t)-\dot{\mathrm{A}}(t) & \dot{\mathrm{P}}(t)-\dot{\mathrm{A}}(t)\end{array}\right)$,

and the propagator is given by

$G\left(t \mid t_{1}\right)=\frac{1}{2}\left(\begin{array}{ll}1+\mathrm{P}\left(t, t_{1}\right)+\mathrm{A}\left(t, t_{1}\right) & 1-\mathrm{P}\left(t, t_{1}\right)+\mathrm{A}\left(t, t_{1}\right) \\ 1-\mathrm{P}\left(t, t_{1}\right)-\mathrm{A}\left(t, t_{1}\right) & 1+\mathrm{P}\left(t, t_{1}\right)-\mathrm{A}\left(t, t_{1}\right)\end{array}\right)$

where

$\mathrm{P}\left(t, t_{1}\right)=\exp \left[\mathrm{P}(t)-\mathrm{P}\left(t_{1}\right)\right]$

$\mathrm{A}\left(t, t_{1}\right)=\int_{t_{1}}^{t} \mathrm{P}(t, s) \dot{A}(s) d s$.

The semigroup property of $G\left(t \mid t_{1}\right)$ follows immediately from the fact that $P\left(t, t_{1}\right)$ and $A\left(t, t_{1}\right)$ satisfy the Markov conditions (A.4).

In the case of definition (2.11) one has

$\dot{\mathrm{P}}(t)=\left.\frac{d}{d s} \rho(s, t)\right|_{s=t^{+}} \leqq 0, \quad \dot{\mathrm{A}}(t)=\left.\frac{d}{d s} \alpha(s, t)\right|_{s=t^{+}}$

which depend on the initial distribution.

In the case of definition (2.12) one finds

$\dot{\mathrm{P}}(t)=\dot{\rho}\left(t, t_{0}\right) / \rho\left(t, t_{0}\right)$

$\dot{A}(t)=\dot{\alpha}\left(t, t_{0}\right)-\dot{\mathrm{P}}(t) \alpha\left(t, t_{0}\right)$

whence

$\mathrm{P}\left(t, t_{1}\right)=\rho\left(t, t_{0}\right) / \rho\left(t_{1}, t_{0}\right)$

$\mathrm{A}\left(t, t_{1}\right)=\alpha\left(t, t_{0}\right)-\mathrm{P}\left(t, t_{1}\right) \alpha\left(t_{1}, t_{0}\right)$,

independent of the initial distribution. It is easy to verify that in this case $\mathrm{P}\left(t, t_{0}\right)=\rho\left(t, t_{0}\right), \mathrm{A}\left(t, t_{0}\right)=\alpha\left(t, t_{0}\right)$ as required by (2.17).
The process is ergodic if $\rho(t,-\infty)=0$ and $\alpha_{\text {as }}(t,-\infty)=$ $a_{\mathrm{as}}(t)$ exist. Asymptotic stationarity requires that $\rho_{\mathrm{as}}\left(t, t_{1}\right)$ and $\alpha_{\mathrm{as}}\left(t, t_{1}\right)$ depend on $t-t_{1}$ only, and are related by

$\left[1-\rho_{\mathrm{as}}\left(t-t_{1}\right)\right] a_{\mathrm{as}}=\alpha_{\mathrm{as}}\left(t-t_{1}\right)$.

In the case of definition (2.11) it follows that

$\mathrm{P}_{\mathrm{as}}\left(t, t_{1}\right)=\exp \left[-\gamma\left(t-t_{1}\right)\right]$

$\mathrm{A}_{\mathrm{as}}\left(t, t_{1}\right)=a_{\mathrm{as}}\left\{1-\exp \left[-\gamma\left(t-t_{1}\right)\right]\right\}$

with $\gamma=-\dot{\rho}_{\text {as }}(0)>0$, which shows the existence of a stationary asymptotic propagator (2.22).

In the case of definition (2.12), on the other hand, $G\left(t \mid t_{0}\right)=R\left(t \mid t_{0}\right)$ becomes singular for $t_{0} \rightarrow-\infty$ because of $\rho\left(t \mid t_{0}\right) \rightarrow 0$, as required by (2.23).

\section{Appendix B. Non-Markov-Gauss Process}

The single-event distribution of a general Gauss process is determined by the mean $a(t)$ and the variance $s(t)$

$p(x t)=[2 \pi s(t)]^{-1 / 2} \exp \left\{-[x-a(t)]^{2} / 2 s(t)\right\}$

and the conditional probability has the form

$R\left(x t \mid y t_{1}\right)=\frac{\exp \left\{-\left[x-\rho\left(t, t_{1}\right) y-\alpha\left(t, t_{1}\right)\right]^{2} / 2 \sigma\left(t, t_{1}\right)\right\}}{\left[2 \pi \sigma\left(t, t_{1}\right)\right]^{1 / 2}}$.

The time evolution of the single-event distribution is given by

$a(t)=\alpha\left(t, t_{1}\right)+\rho\left(t, t_{1}\right) a\left(t_{1}\right)$

$s(t)=\sigma\left(t, t_{1}\right)+\rho^{2}\left(t, t_{1}\right) s\left(t_{1}\right)$.

The conditional probabilities (B.2) do not form a semigroup except in the Markov case

$\rho\left(t, t_{1}\right)=\rho(t, s) \rho\left(s, t_{1}\right)$

$\alpha\left(t, t_{1}\right)=\alpha(t, s)+\rho(t, s) \alpha\left(s, t_{1}\right)$

$\sigma\left(t, t_{1}\right)=\sigma(t, s)+\rho^{2}(t, s) \sigma\left(s, t_{1}\right)$.

The generator of the semigroup corresponding to Equation (2.11) is the "linear" Fokker-Planck operator

$\Gamma(t)=\frac{\partial}{\partial x}\left[-\dot{\rho}(t) x-\dot{\alpha}(t)+\frac{1}{2} \dot{\sigma}(t) \frac{\partial}{\partial x}\right]$

where

$$
\begin{aligned}
& \dot{\rho}(t)=\left.\frac{d}{d s} \rho(s, t)\right|_{s=t^{+}} \\
& \dot{\alpha}(t)=\left.\frac{d}{d s} \alpha(s, t)\right|_{s=t^{+}} \\
& \dot{\sigma}(t)=\left.\frac{d}{d s} \sigma(s, t)\right|_{s=t^{+}} .
\end{aligned}
$$


$\Gamma(t)$ generates the general Gauss-Markov semigroup (see Ref. 16)

$G\left(x t \mid y t_{1}\right)=\frac{\exp \left\{-\left[x-\mathrm{P}\left(t, t_{1}\right) y-\mathrm{A}\left(t, t_{1}\right)\right]^{2} / 2 \Sigma\left(t, t_{1}\right)\right\}}{\left[2 \pi \Sigma\left(t, t_{1}\right)\right]^{1 / 2}}$

where

$\mathrm{P}\left(t, t_{1}\right)=\exp \left[\rho(t)-\rho\left(t_{1}\right)\right]$

$\mathrm{A}\left(t, t_{1}\right)=\int_{t_{1}}^{t} \mathrm{P}(t, s) \dot{\alpha}(s) d s$

$\Sigma\left(t, t_{1}\right)=\int_{t_{1}}^{t} \mathrm{P}^{2}(t, s) \dot{\sigma}(s) d s$.

Calculation of the $p_{0}$-independent generator according to Equation (2.12) would require the inverse of $R\left(x t \mid y t_{1}\right)$ and is not carried out here.

For an ergodic process, $\rho(t,-\infty)=0, \alpha(t,-\infty)=a_{\mathrm{as}}(t)$ and $\sigma(t,-\infty)=s_{\text {as }}(t)$ exist. Asymptotic stationarity requires that $\rho_{\text {as }}\left(t, t_{1}\right), \alpha_{\text {as }}\left(t, t_{1}\right)$ and $\sigma_{\text {as }}\left(t, t_{1}\right)$ depend on $t-t_{1}$ only, and satisfy

$\left[1-\rho_{\mathrm{as}}\left(t-t_{1}\right)\right] a_{\mathrm{as}}=\alpha_{\mathrm{as}}\left(t-t_{1}\right)$

$\left[1-\rho_{\mathrm{as}}^{2}\left(t-t_{1}\right)\right] s_{\mathrm{as}}=\sigma_{\mathrm{as}}\left(t-t_{1}\right)$.

As a consequence,

$\mathrm{P}_{\mathrm{as}}\left(t, t_{1}\right)=\exp \left[-\gamma\left(t-t_{1}\right)\right]$

$\mathrm{A}_{\mathrm{as}}\left(t, t_{1}\right)=a_{\mathrm{as}}\left\{1-\exp \left[-\gamma\left(t-t_{1}\right)\right]\right\}$

$\Sigma_{\text {as }}\left(t, t_{1}\right)=s_{\text {as }}\left\{1-\exp \left[-2 \gamma\left(t-t_{1}\right)\right]\right\}$ where $\gamma=-\dot{\rho}_{\text {as }}(0)>0$, which shows the existence of a stationary asymptotic propagator (2.22).

\section{References}

1. Zwanzig, R.: Phys. Rev. 124, 983 (1961)

2. Mori, H., Fujisaka, H.: Progr. Theor. Phys. 49, 764 (1973)

3. Haake, F.: Springer Tracts in Modern Physics 66, 98 (1973)

4. Haken, H.: Rev. Mod. Phys. 47, 67 (1975)

5. Mori, H.: In: Proceedings of the International Symposium of Synergetics, edited by H. Haken, Stuttgart: Teubner 1972

6. Haake, F.: Z. Physik 223, 353 (1969); 227, 179 (1969)

7. Kenkre, V. M., Knox, R. S.: Phys. Rev. B 9, 5279 (1974)

8. Hynes, J.T., Kapral, R., Weinberg, M.: Physica 80 A, 105 (1975)

9. Pomeau, Y., Résibois, P.: Phys. Rep. 19C, 63 (1975)

10. Lebowitz, J.L., Rubin, E.: Phys. Rev. 131, 2381 (1963)

11. Doob, J.L.: Stochastic Processes, chapt. VI, New York: Wiley 1953

12. Chang, E.L., Mazo, R. M., Hynes, J. T.: Mol. Phys. 28, 997 (1974)

13. Adelman, S. A.: J. chem. Phys. 64, 124 (1976)

14. Haake, F.: Z. Physik 223, 364 (1969)

15. Agarwal, G.S.: Z. Physik 258, 401 (1972)

16. Hänggi, P., Thomas, H.: Z. Physik B 22, 295 (1975)

P. Hänggi

H. Thomas

Institut für Physik

der Universität Basel

K.lingelbergstrasse 82

CH-4056 Basel

Switzerland 